\title{
Perioperative alectinib in a patient with locally advanced anaplastic lymphoma kinase positive non-small cell lung cancer (NSCLC): a case report
}

\author{
Ping Yue ${ }^{1 \#}$, Shengxiong Zhang ${ }^{1 \#}$, Ling Zhou ${ }^{1,2}$, Jie Xiang $^{3}$, Shengguang Zhao ${ }^{4}$, Xiaoyan Chen $^{5}$, Lei Dong ${ }^{5}$, \\ Wenjie Yang ${ }^{6}$, Yi Xiang ${ }^{1,2}$ \\ ${ }^{1}$ Department of Respiratory and Critical Care Medicine, Ruijin Hospital, Shanghai Jiaotong University School of Medicine, Shanghai, China; \\ ${ }^{2}$ Institute of Respiratory Diseases, Shanghai Jiaotong University School of Medicine, Shanghai, China; ${ }^{3}$ Department of Thoracic Surgery, Ruijin \\ Hospital, Shanghai Jiaotong University School of Medicine, Shanghai China; ${ }^{4}$ Department of Radiotherapy, Ruijin Hospital, Shanghai Jiaotong \\ University School of Medicine, Shanghai, China; ${ }^{5}$ Department of Pathology, Ruijin Hospital, Shanghai Jiaotong University School of Medicine, \\ Shanghai, China; ${ }^{6}$ Department of Radiology, Ruijin Hospital, Shanghai Jiaotong University School of Medicine, Shanghai, China \\ \#These authors contributed equally to this work. \\ Correspondence to: Yi Xiang. Department of Respiratory and Critical Care Medicine, Institute of Respiratory Diseases, Ruijin Hospital, Shanghai \\ Jiaotong University School of Medicine, Shanghai, China. Email: xiangyiht@163.com.
}

\begin{abstract}
We reported a case of locally advanced anaplastic lymphoma kinase (ALK) positive nonsmall cell lung cancer (NSCLC) patient who received neoadjuvant alectinib therapy. Enhanced computed tomography (CT) scan was performed after the first cycle of alectinib therapy to evaluate the efficacy of neoadjuvant alectinib. Surprisingly, the tumor shrunk 42.2\% after one cycle treatment. Partial remission (PR) was achieved without any side effects, although the tumor stage didn't degrade. Then right upper lobectomy and mediastinal lymph node dissection by video assistant thoracoscopic surgery (VATS) were successfully performed after multi-disciplinary team meeting with the department of respiratory, thoracic surgery, radiotherapy (RT), pathology and radiology. Pathologic evaluation about tumor was assessed by hematoxylin and eosin staining. However, the residual viable tumor cells were $15 \%$, which indicated that major pathologic response (MPR) was not achieved. Next, continually adjuvant alectinib and RT were given because mediastinal station 4R lymphadenectomy excluded with serious tissue adhesion and MPR status was not met. In this case, we presented neoadjuvant alectinib therapy was feasible and well tolerated in locally advanced ALK positive NSCLC, inspiring clinical studies to further assess its clinical implication in treating patients with locally advanced ALK-positive NSCLC. And we also discussed the necessary time of neoadjuvant and adjuvant alectinib in advanced ALK-positive NSCLC.
\end{abstract}

Keywords: Locally advanced non-small cell lung cancer (locally advanced NSCLC); anaplastic lymphoma kinase (ALK); perioperative target therapy; alectinib; case report

Submitted Apr 13, 2021. Accepted for publication Jun 16, 2021.

doi: $10.21037 /$ tcr-21-642

View this article at: https://dx.doi.org/10.21037/tcr-21-642

\section{Introduction}

About $5 \%$ of patients of all non-small cell lung cancer (NSCLC) have anaplastic lymphoma kinase (ALK) gene rearrangement, especially in those who have never smoked or have a history of light smoking, as well as in those with adenocarcinomas (1). Locally advanced NSCLC has always been considered as a highly heterogeneous clinical issue (2).

^ ORCID: Ping Yue, 0000-0002-9996-1017; Yi Xiang, 0000-0002-0724-6359. 
Studies have proved that adjuvant tyrosine kinase inhibitors (TKIs) prolong significantly longer disease-free survival (DFS) compared with that with chemotherapy in patients with resected early/locally advanced stage of NSCLC who harbor epidermal growth factor receptor (EGFR) mutations (3-5). There are also series of cases have reported that neoadjuvant crizotinib may be feasible and well tolerated in locally advanced ALK positive patients $(6,7)$. Alectinib, as the secondgeneration TKI, is a highly selective ALK inhibitor with high central nervous system (CNS) penetration. Alectinib showed superior efficacy and lower toxicity than crizotinib in primary ALK-positive patients. Moreover, Alectinib showed clinical activity in patients with ALK-positive NSCLC and who have progressed on crizotinib (8). In phase III ALEX Study (9), 303 untreated ALK-positive advanced NSCLC patients were randomized to receive treatment with alectinib $(n=152)$ or crizotinib $(\mathrm{n}=151)$. The median progression free survival (PFS) was significantly longer with alectinib (34.8 months) than with crizotinib (10.9 months). Another phase 3 randomized trial (J-ALEX) assessed first-line therapy with alectinib versus crizotinib in 207 Japanese patients, showing that alectinib was superior than crizotinib in ALK inhibitor-naive patients with ALK-positive NSCLC (10).

In addition, Zhang et al. had first reported a clinically successful case which illustrated neoadjuvant alectinib for a metastatic lung adenocarcinoma patient staging as cT3N2M0 IIIB was feasible (11). After 56 days (two cycles) of neoadjuvant alectinib at a dosage of $600 \mathrm{mg}$ twice per day, a partial response was achieved with a tumor shrinkage of $47 \%$. Then Lobectomy and systemic lymphadenectomy were successfully performed without any severe in-hospital complications. Moreover, MPR was achieved with only $7 \%$ remaining tumor cells in this case. Owing to these improvements demonstrated in advanced NSCLC, we supposed alectinib in early/locally advanced resected stage of the NSCLC could acquire better efficacy than chemotherapy. However, there are rare studies about alectinib in locally advanced NSCLC patients' neoadjuvant therapy. In this article, we reported a clinically efficacious locally advanced ALK positive case involving neoadjuvant alectinib. We present the following case in accordance with the CARE reporting checklist (available at https://dx.doi.org/10.21037/tcr-21-642).

\section{Case presentation}

A 51 years old nonsmoking man, had a routine physical examination because of right foot numbness. He had a history of hypertension and had oral drugs to control it. He and his family did not have any specific history of malignant tumor. Chest X-ray showed a high-density nodule located in the right upper lobe. Then enhanced chest computed tomography (CT) revealed a nodule measured $31 \mathrm{~mm}$ in diameter and accompanied by enlarged mediastinal lymph nodes with discrete 4R and 10R (Figure 1). Transbronchial needle aspiration was performed for stations $4 \mathrm{R}$ and $10 \mathrm{R}$ through endobronchial ultrasound. And no signs of distant metastasis were confirmed with enhanced brain magnetic resonance imaging (MRI) and positron emission tomography-computed tomography (PET-CT). The pathologic evaluation showed a metastatic lung adenocarcinoma and the clinical stage was IIIA (cT2N2M0). Immunohistochemistry (IHC) revealed positive staining of cytokeratin AE1/AE3, thyroid transcription factor-1 (TTF-1), Napsin A, and ALK fusion status was positive assessed by IHC with a monoclonal antibody (D5F3, VentanaRoche Diagnostics, Mannheim, Germany) and fluorescence in situ hybridization (FISH) (Vysis ALK Break Apart FISH Probe Kit; Abbott, IL, USA). The percent of rearranged cells in FISH was $45.7 \%$ (Figure 2).

Surgery is not a standard therapy in stage III N2 NSCLC patients. In patients with genomic defined NSCLC, a currently investigated option is the use of TKI in neoadjuvant setting. Therefore, an immediate surgery was not performed after multi-disciplinary team consultation by the department of respiratory, thoracic surgery, radiotherapy (RT), pathology and radiology. After acquiring informed consent from the patient, we offered neoadjuvant alectinib at a dosage of $600 \mathrm{mg}$ twice per day from April 28, 2020. Naturally, the tumor shrunk $42.2 \%$ after one cycle (28 days) therapy of alectinib, which was confirmed by CT and PET-CT scan (Figures 1,3), although the clinical stage was also IIIA (cT1N2M0). And this patient was evaluated as partial remission (PR) by Response Evaluation Criteria in Solid Tumors (RECIST) version 1.1. Moreover, the patient did not show any side effects with alectinib treatment.

Then right upper lobectomy with mediastinal lymph node dissection by VATS were performed after 45 days (2020/4/28-2020/6/11) treatment of alectinib. The mediastinal lymph node station $4 \mathrm{R}$ was inoperative because of high surgical risk caused by severe tissue adhesion. The patient was discharged 5 days after operation, without any in-hospital and surgical complications. Pathologic evaluation about tumor was assessed by hematoxylin and eosin staining and IHC (Figure 4), however MPR was not achieved (the residual viable tumor cells were $15 \%$ ). And the patient continually received adjuvant alectinib $(600 \mathrm{mg}$ oral BID) a week after surgery. The patient did not make 
nodule
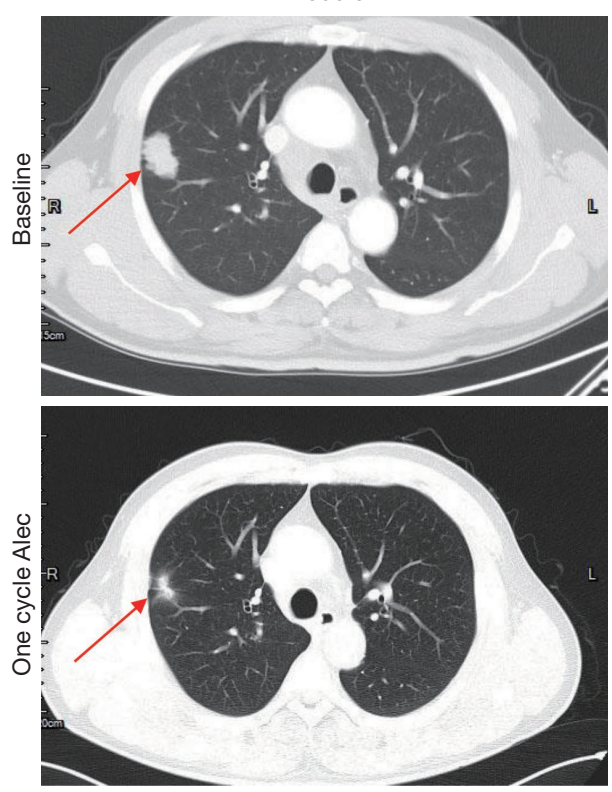

$4 \mathrm{R}$
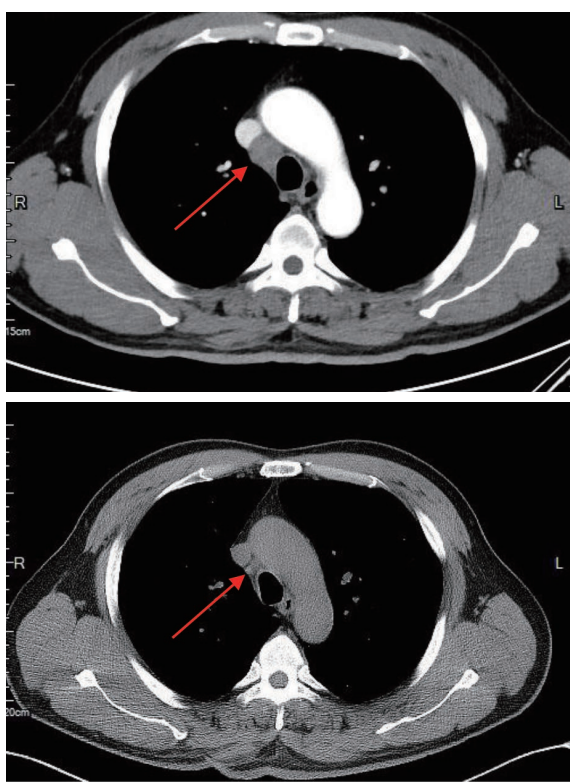

$10 \mathrm{R}$
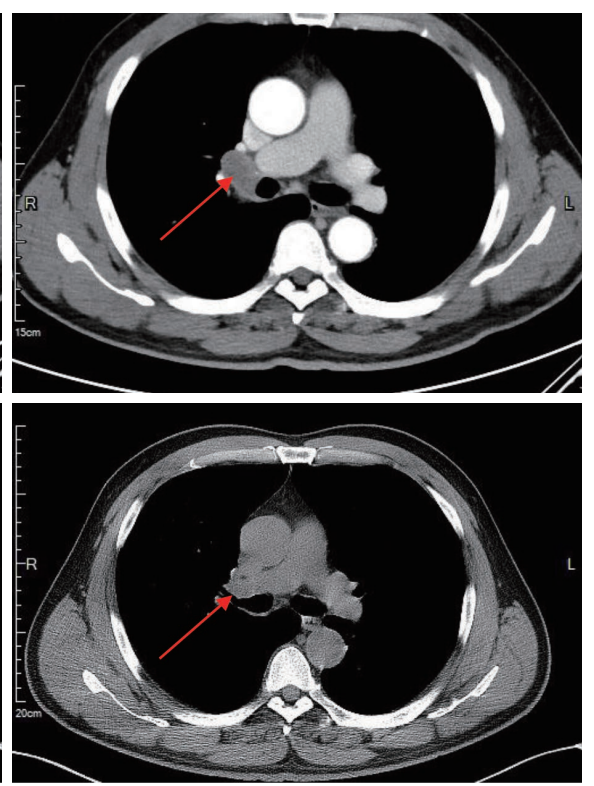

Figure 1 The CT scan of baseline and after one cycle of alectinib (the red arrows stand for primary lung cancer, 4R lymph node and 10R lymph node respectively). CT, computed tomography.

routine follow-up due to the epidemic of COVID-19. Thus, the second cycle of multi-disciplinary team consultation was performed owing to the patients without systematic mediastinal lymph node dissection (station $4 \mathrm{R}$ excluded) and MPR status was not met. We recommended the sequential radiotherapy (RT) combined with alectinib therapy, considering the patient physical performance and the willingness. The postoperative radiotherapy (PORT) containing areas of the removed tumor and $4 \mathrm{R}$ lymph nodes was given at $50 \mathrm{~Gy} / 25 \mathrm{Fx}$ and the duration was 1 month. No recurrence was observed after the last follow-up (Figure 5). And the patient did not complain any discomfort. The whole course of treatment was showed in Figure 6.

All procedures performed in studies involving human participants were in accordance with the ethical standards of the institutional and/or national research committee(s) and with the Helsinki Declaration (as revised in 2013). Written informed consent was obtained from the patient.

\section{Discussion}

We here report an ALK positive case involving the advantage of alectinib during perioperative period. Together with another report by Zhang (11) et al., which reported neoadjuvant alectinib was clinically feasible. These data supported that alectinib would be effective and safe in patients with locally advanced ALK positive NSCLC. According to ALEX study, alectinib showed superior efficacy and lower toxicity in primary treatment of ALKpositive advanced NSCLC compared with crizotinib (12). Whether neoadjuvant alectinib is appropriated and safe is unknown so far. In our case, the tumor response achieved PR after one cycle of alectinib (600 mg, oral, BID, 30 days) and then lobectomy and mediastinal lymph node dissection were performed.

However, the patient did not achieve MPR status with almost two cycles of neoadjuvant alectinib, the residual viable tumor cells were $15 \%$. In CTONG 1103 (EMERGING) study, the erlotinib arm consisted of neoadjuvant erlotinib $150 \mathrm{mg} / \mathrm{d}$ (42 days, oral) followed by adjuvant erlotinib postoperatively for 12 months or until disease progression or unacceptable toxicity (13), which R0 resection and lymph node downstaging were more than the neoadjuvant chemotherapy arm $(73 \%$ and $10.8 \%$ vs. $63 \%$ and $2.9 \%$ ), demonstrating two cycles of neoadjuvant TKI therapy were necessary. However, in the case published by Zhang et al. (11), the patient completed two cycles of alectinib achieving MPR with only $7 \%$ of residual viable tumor cells. One possible explanation to that is the ORR with TKIs in the neoadjuvant therapy was lower than that in 


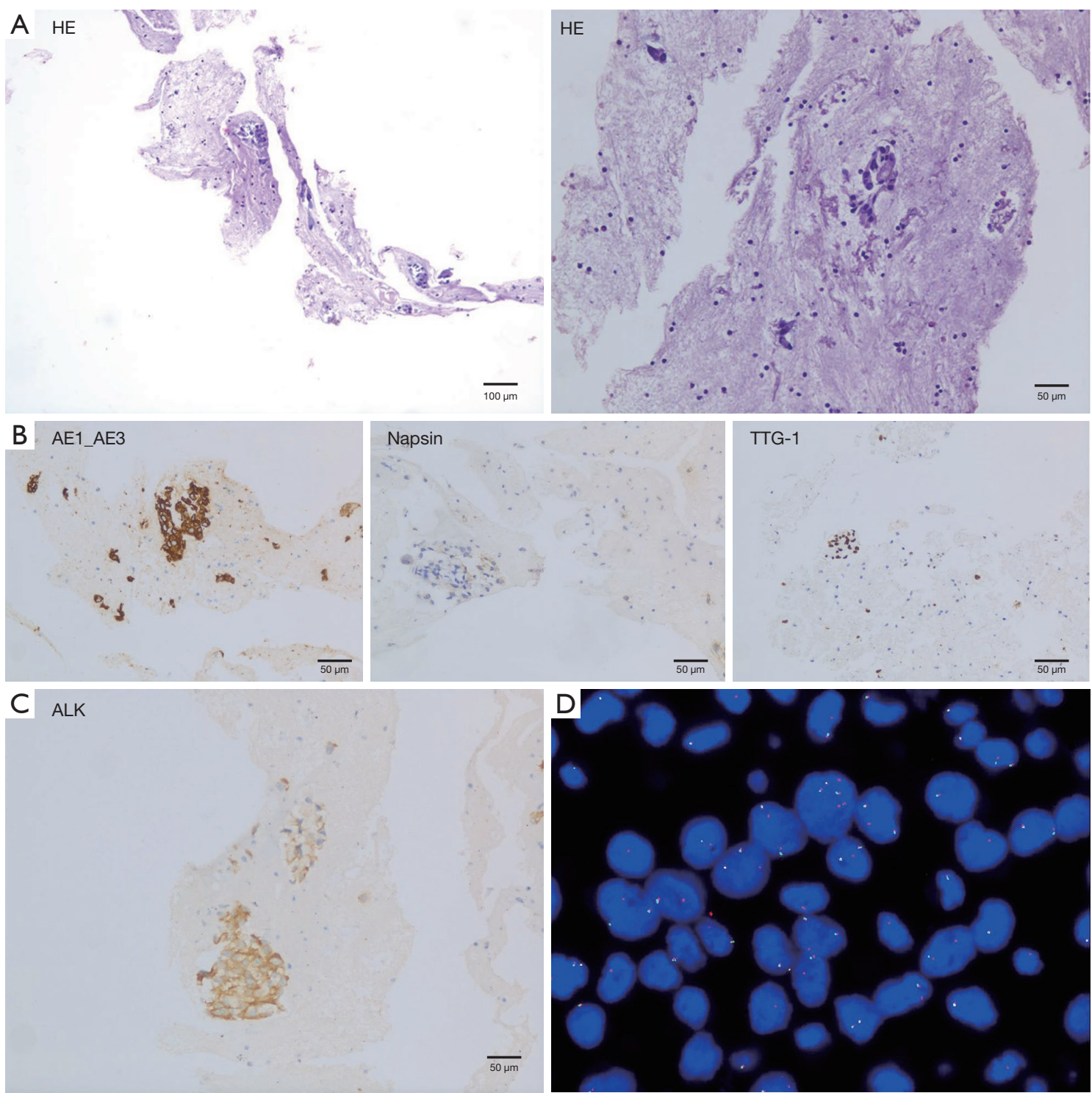

Figure 2 Integrated pathological validation of lung adenocarcinoma harboring $A L K$ gene rearrangement. (A) $\mathrm{HE}$ staining, 10× and 20×. (B) Immunohistochemistry, including cytokeratin AE1/AE3 (AE1/AE3), Napsin, TTF-1, 20×. (C) ALK fusion, Immunohistochemistry, 20× (D5F3; Ventana-Roche). (D) ALK fusion, FISH (Vysis ALK Break Apart FISH Probe Kit; Abbott, IL, USA). ALK, anaplastic lymphoma kinase; HE, hematoxylin-eosin; TTF-1, thyroid transcription factor-1; FISH, fluorescence in situ hybridization.

advanced NSCLC. In addition, the limited duration of drug exploration was another reason (13-15). Further studies are needed to verify the necessity of extended neoadjuvant treatment of TKIs.

Moreover, the duration of adjuvant alectinib was controversial. According to the ADJUVANT, RADIANT, EVEN and SELECT trials, patients received gefitinib or erlotinib for almost 24 months, while patients were administrated osimertinib for 3 years in ADAURA trial.
And all above studies have shown a significantly longer DFS in patients who received TKIs than the control groups at 24 months $(4,5,16-18)$. In the SELECT trial, $65 \%$ patients with recurrence retreated with erlotinib and only one patient who acquired T790m didn't take erlotinib again, which suggested the cause of recurrence was stopping the suppressive erlotinib rather than truly acquiring resistance to erlotinib (17). In the ADJUVANT trail, 3-year DFS rate in the intention-to-treat population was 34\% (95\% CI: 
nodule
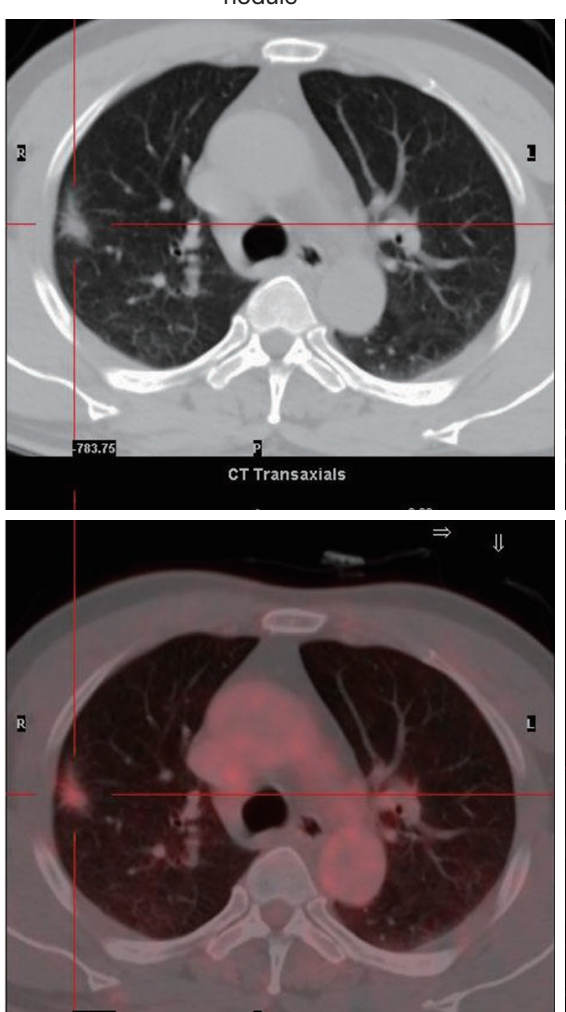

LN 4 R
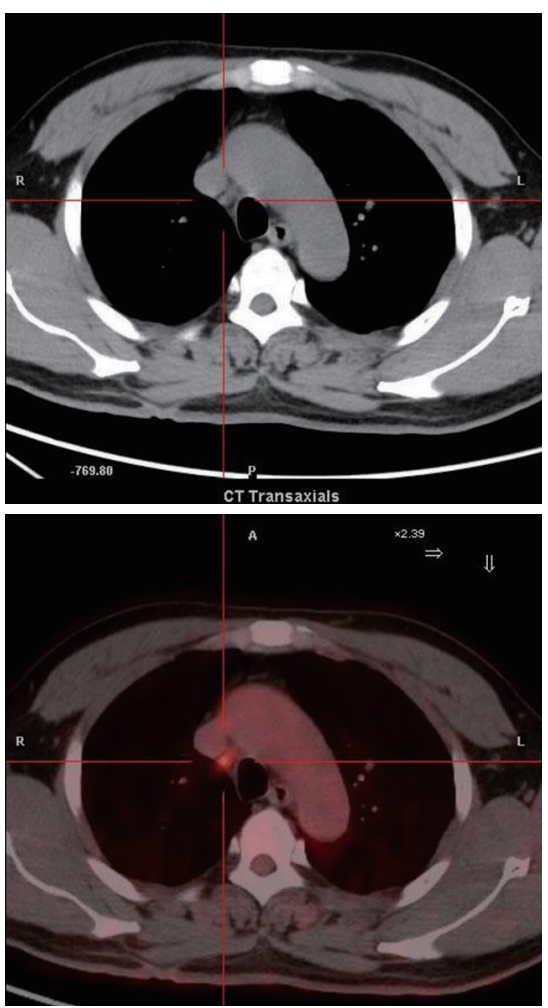

LN $10 \mathrm{R}$
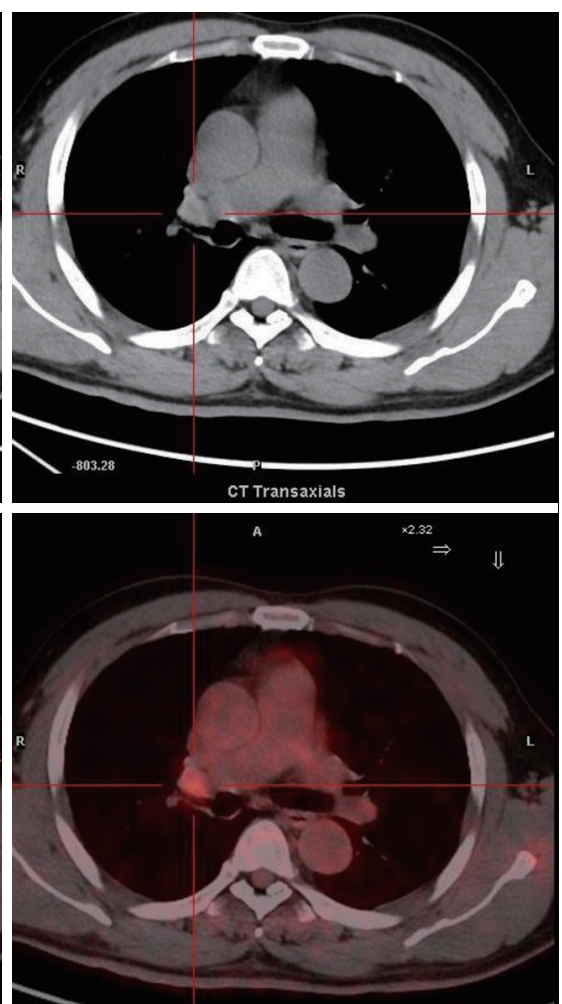

Figure 3 The PET-CT scan assessment of one cycle alectinib. LN, lymph node; PET-CT, positron emission tomography-computed tomography.
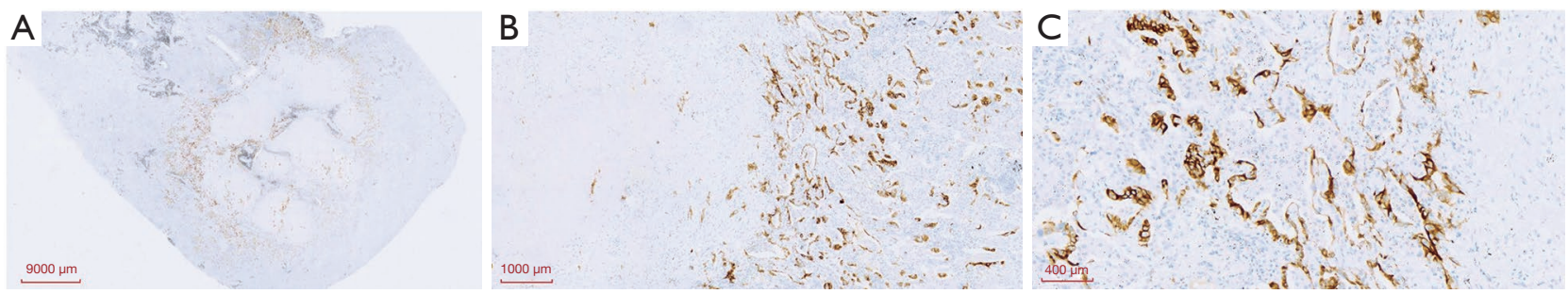

Figure 4 The $A L K$ gene rearrangement of surgical specimens. (A) 5×, (B) 40×, (C) 100×. ALK, anaplastic lymphoma kinase.

24-45) in the gefitinib group and $27 \%$ (95\% CI: 16-38) in the vinorelbine plus cisplatin group (HR 0.74, 95\% CI: $0.42-1.32 ; \mathrm{P}=0.37)$. The non-significant difference in DFS proportion between two arms suggested gefitinib may delay but not necessarily prevent recurrences (4). In general, it means that 2 years adjuvant targeted therapy are not enough, so we consider 3 years treatment of alectinib rather than 2 years may be necessary although the optimal duration of adjuvant TKI therapy will remain undefined.

Still, there are several limitations in this case. First, we did not perform systemic lymphadenectomy resection due to intraoperative tissue adhesions. Neoadjuvant targeted therapy may increase the difficulty of surgery, indicating that we should pay more attention to the safety of surgery in future preoperative targeted therapy research. Second, although this patient did not meet the criteria of R0 resection, PORT was performed nearly 2 months after the surgery because of the epidemic of COVID-19. Although PORT in patients with a completely resected stage III-N2 NSCLC showed poor benefits on DFS, according to the 

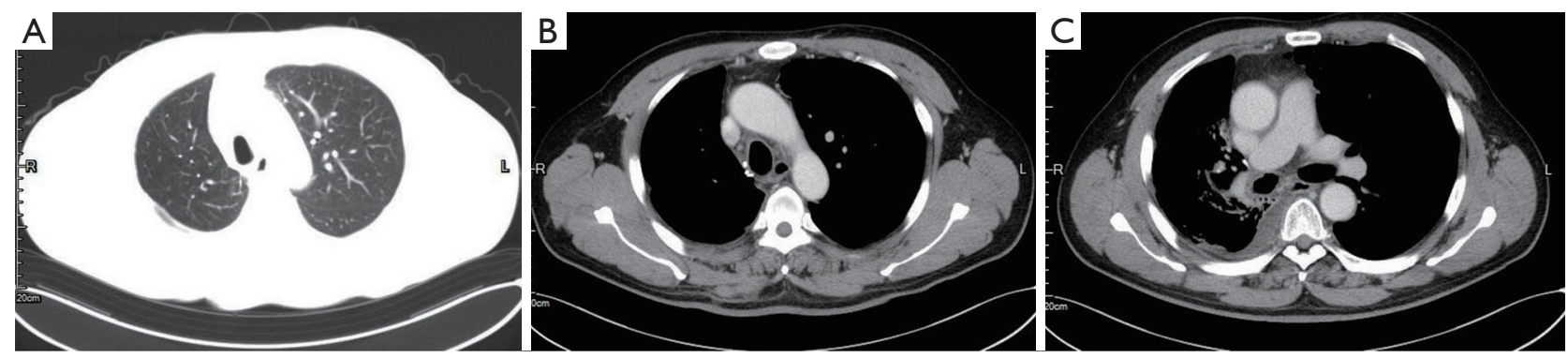

Figure 5 The CT scan of the patient's last followed up on December 8, 2020. CT, computed tomography.

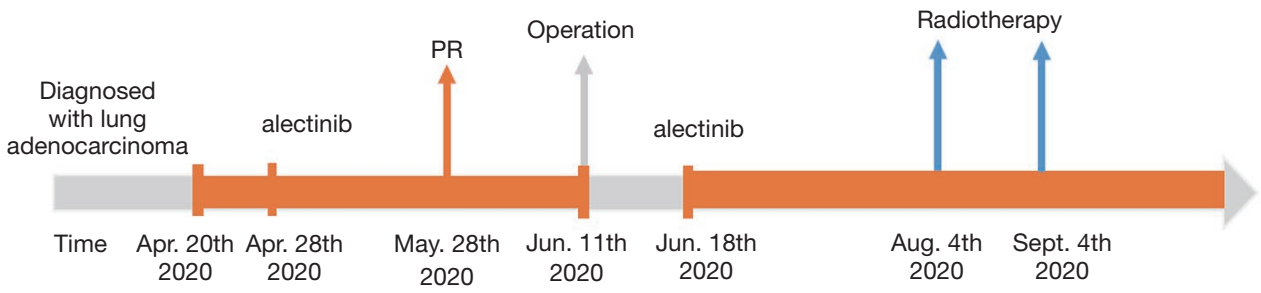

Figure 6 The timeline of treatment. PR, partial regression.

results of the randomized LungART trial (19). However, PORT is an alternative for patients with resectable stage IIIA disease recommended by NCCN guideline (Version 3.2020) with the evidence showed that PORT improve survival benefit significantly in patients with mediastinal nodal involvement (pN2) (20). Whether RT combined TKIs could improve the overall survival is unknown. A study enrolled ten patients with stage IV NSCLC harboring EGFR mutations who received first-line EGFR-TKI combined with thoracic RT showed concurrent EGFR-TKI plus RT as the first-line treatment prolonged the control of lung lesion, which 1-year PFS rate was $57.1 \%$, median PFS was 13 months, and median time to progression of irradiated lesion (iTTP) was 20.5 months (21). However, whether alectinib plus PORT could achieve benefit over the toxicity, especially the pN2-IIIA patients, needs more trails to demonstrate.

In conclusion, according to data addressed using TKIs in the locally advanced NSCLC patients with druggable targets, we reported a case which demonstrated that alectinib was efficient and safe as a neoadjuvant treatment in local advanced ALK positive NSCLC patients. At present, there is a recruiting clinical trial comparing the efficiency of adjuvant alectinib with adjuvant platinumbased chemotherapy in patients with ALK positive NSCLC (NCT03456076). We also need to try neoadjuvant alectinib therapy based on previous data and experience from TKIs in the locally advanced NSCLC patients to further assess the role of neoadjuvant alectinib in locally advanced ALK positive NSCLC.

\section{Acknowledgments}

We thank the patient who participated in this study.

Funding: This work was approved by grants from National Natural Science Foundation of China (81672271) and Shanghai Key Discipline for Respiratory Diseases (2017ZZ02014).

\section{Footnote}

Reporting Checklist: The authors have completed the CARE reporting checklist. Available at https://dx.doi. org/10.21037/tcr-21-642

Peer Review File: Available at https://dx.doi.org/10.21037/ tcr-21-642

Conflicts of Interest: All authors have completed the ICMJE uniform disclosure form (available at https://dx.doi. org/10.21037/tcr-21-642). The authors have no conflicts of interest to declare.

Ethical Statement: The authors are accountable for all 
aspects of the work in ensuring that questions related to the accuracy or integrity of any part of the work are appropriately investigated and resolved. All procedures performed in studies involving human participants were in accordance with the ethical standards of the institutional and/or national research committee(s) and with the Helsinki Declaration (as revised in 2013). Written informed consent was obtained from the patient for publication of this case report and any accompanying images. A copy of the written consent is available for review by the editorial office of this journal.

Open Access Statement: This is an Open Access article distributed in accordance with the Creative Commons Attribution-NonCommercial-NoDerivs 4.0 International License (CC BY-NC-ND 4.0), which permits the noncommercial replication and distribution of the article with the strict proviso that no changes or edits are made and the original work is properly cited (including links to both the formal publication through the relevant DOI and the license). See: https://creativecommons.org/licenses/by-nc-nd/4.0/.

\section{References}

1. Kwak EL, Bang YJ, Camidge DR, et al. Anaplastic lymphoma kinase inhibition in non-small-cell lung cancer. N Engl J Med 2010;363:1693-703.

2. Rigas JR, Kelly K. Current treatment paradigms for locally advanced non-small cell lung cancer. J Thorac Oncol 2007;2 Suppl 2:S77-85.

3. Cheng H, Li XJ, Wang XJ, et al. A meta-analysis of adjuvant EGFR-TKIs for patients with resected non-small cell lung cancer. Lung Cancer 2019;137:7-13.

4. Zhong WZ, Wang Q, Mao WM, et al. Gefitinib versus vinorelbine plus cisplatin as adjuvant treatment for stage II-IIIA (N1-N2) EGFR-mutant NSCLC (ADJUVANT/ CTONG1104): a randomised, open-label, phase 3 study. Lancet Oncol 2018;19:139-48.

5. Wu YL, Tsuboi M, He J, et al. Osimertinib in Resected EGFR-Mutated Non-Small-Cell Lung Cancer. N Engl J Med 2020;383:1711-23.

6. Zhang C, Li SL, Nie Q, et al. Neoadjuvant Crizotinib in Resectable Locally Advanced Non-Small Cell Lung Cancer with ALK Rearrangement. J Thorac Oncol 2019;14:726-31.

7. Tian Y, Huang J, Li C, et al. Perioperative crizotinib in a patient with stage IIIB ALK-positive non-small cell lung cancer: a case report. Ann Transl Med 2020;8:770.
8. Shaw AT, Gandhi L, Gadgeel S, et al. Alectinib in ALKpositive, crizotinib-resistant, non-small-cell lung cancer: a single-group, multicentre, phase 2 trial. Lancet Oncol 2016;17:234-42.

9. Camidge DR, Dziadziuszko R, Peters S, et al. Updated Efficacy and Safety Data and Impact of the EML4-ALK Fusion Variant on the Efficacy of Alectinib in Untreated ALK-Positive Advanced Non-Small Cell Lung Cancer in the Global Phase III ALEX Study. J Thorac Oncol 2019;14:1233-43.

10. Hida T, Nokihara H, Kondo $M$, et al. Alectinib versus crizotinib in patients with ALK-positive non-small-cell lung cancer (J-ALEX): an open-label, randomised phase 3 trial. Lancet 2017;390:29-39.

11. Zhang C, Yan LX, Jiang BY, et al. Feasibility and Safety of Neoadjuvant Alectinib in a Patient With ALK-Positive Locally Advanced NSCLC. J Thorac Oncol 2020;15:e95-9.

12. Peters S, Camidge DR, Shaw AT, et al. Alectinib versus Crizotinib in Untreated ALK-Positive Non-Small-Cell Lung Cancer. N Engl J Med 2017;377:829-38.

13. Zhong WZ, Chen KN, Chen C, et al. Erlotinib Versus Gemcitabine Plus Cisplatin as Neoadjuvant Treatment of Stage IIIA-N2 EGFR-Mutant Non-Small-Cell Lung Cancer (EMERGING-CTONG 1103): A Randomized Phase II Study. J Clin Oncol 2019;37:2235-45.

14. Zhong W, Yang X, Yan H, et al. Phase II study of biomarker-guided neoadjuvant treatment strategy for IIIA-N2 non-small cell lung cancer based on epidermal growth factor receptor mutation status. J Hematol Oncol 2015;8:54.

15. Takeda M, Okamoto I, Nakagawa K. Survival outcome assessed according to tumor response and shrinkage pattern in patients with EGFR mutation-positive nonsmall-cell lung cancer treated with gefitinib or erlotinib. J Thorac Oncol 2014;9:200-4.

16. Kelly K, Altorki NK, Eberhardt WE, et al. Adjuvant Erlotinib Versus Placebo in Patients With Stage IBIIIA Non-Small-Cell Lung Cancer (RADIANT): A Randomized, Double-Blind, Phase III Trial. J Clin Oncol 2015;33:4007-14.

17. Pennell NA, Neal JW, Chaft JE, et al. SELECT: A Phase II Trial of Adjuvant Erlotinib in Patients With Resected Epidermal Growth Factor ReceptorMutant Non-Small-Cell Lung Cancer. J Clin Oncol 2019;37:97-104.

18. Yue D, Xu S, Wang Q, et al. Erlotinib versus vinorelbine plus cisplatin as adjuvant therapy in Chinese patients with 
stage IIIA EGFR mutation-positive non-small-cell lung cancer (EVAN): a randomised, open-label, phase 2 trial. Lancet Respir Med 2018;6:863-73.

19. Le Pechoux C, Pourel N, Barlesi F, et al. An international randomized trial, comparing postoperative conformal radiotherapy (PORT) to no PORT, in patients with completely resected non-small cell lung cancer (NSCLC) and mediastinal N2 involvement: Primary end-point analysis of LungART (IFCT0503, UK NCRI, SAKK) NCT00410683. Ann Oncol

Cite this article as: Yue $\mathrm{P}$, Zhang S, Zhou L, Xiang J, Zhao S, Chen X, Dong L, Yang W, Xiang Y. Perioperative alectinib in a patient with locally advanced anaplastic lymphoma kinase positive non-small cell lung cancer (NSCLC): a case report. Transl Cancer Res 2021;10(8):3856-3863. doi: 10.21037/tcr-21-642
2020;31:1142-215.

20. Corso CD, Rutter CE, Wilson LD, et al. Re-evaluation of the role of postoperative radiotherapy and the impact of radiation dose for non-small-cell lung cancer using the National Cancer Database. J Thorac Oncol 2015;10:148-55.

21. Zheng L, Wang Y, Xu Z, et al. Concurrent EGFR-TKI and Thoracic Radiotherapy as First-Line Treatment for Stage IV Non-Small Cell Lung Cancer Harboring EGFR Active Mutations. Oncologist 2019;24:1031-e612. 